\title{
Multivariate analysis of metastasis-related risk factors for patients with gastroenteropancreatic neuroendocrine tumors based on clinicopathological and endoscopic features
}

\author{
CAIYUN TANG ${ }^{1,3^{*}}$, LINGQI GONG ${ }^{2 *}$, WENLI ZOU ${ }^{2 *}$, JIE ZHANG $^{2}$, YUQIAN ZHOU ${ }^{2}$, \\ XIAOPING WU ${ }^{2}$, FANGGEN LU ${ }^{2}$, CHUNHUI OUYANG ${ }^{2}$ and XIAOWEI LIU ${ }^{1}$ \\ ${ }^{1}$ Department of Gastroenterology, Xiangya Hospital of Central South University, Changsha, \\ Hunan 410008; ${ }^{2}$ Department of Gastroenterology, The Second Xiangya Hospital of \\ Central South University, Changsha, Hunan 410011, P.R. China
}

Received April 28,2016; Accepted July 13, 2016

DOI: $10.3892 /$ or.2016.5170

\begin{abstract}
Gastroenteropancreatic neuroendocrine tumors (GEP-NETs) are relatively uncommon. Unfortunately, epidemiological studies on the incidence of GEP-NETs worldwide have reported a marked increase in the detection of these tumors. Although they often exhibit relatively indolent clinical courses, GEP-NETs have the potential for lethal progression, especially in patients who present with advanced disease. Early detection and surgical removal is currently the only reliable curative treatment for GEP-NET patients. The objective of this study was to analyze the clinicopathological characteristics of GEP-NETs and explore the metastasis-related risk factors of patients with GEP-NETs. One hundred and forty-six patients diagnosed pathologically with GEP-NETs from January 2001 to January 2015 at the Second Xiangya Hospital of Central South University were retrospectively evaluated. We retrieved and analyzed information concerning clinical characteristics and metastasis-related risk factors, and used Chi-square test and logistic regression analysis to analyze the clinicopathological characteristics of GEP-NETs and explore the association between tumor metastasis and possible related risk
\end{abstract}

Correspondence to: Professor Xiaowei Liu, Department of Gastroenterology, Xiangya Hospital of Central South University, 87 Xiangya Road, Changsha, Hunan 410008, P.R. China

E-mail: liuxw@csu.edu.cn

Dr Chunhui Ouyang, Department of Gastroenterology, The Second Xiangya Hospital of Central South University, 139 Middle Renmin Road, Changsha, Hunan 410011, P.R. China

E-mail: xiaohuioych@126.com

Present address: ${ }^{3}$ Geriatrics Department, Yiyang Central Hospital, Yiyang, Hunan 413000, P.R. China

*Contributed equally

Key words: gastroenteropancreatic neuroendocrine tumors, carcinoid, lymphatic vessel density, multivariate analysis factors. The results revealed that the most common clinical manifestations were abdominal pain $(n=88)$, alteration in the character of stool $(n=58)$ and melaena $(n=33)$. Rectum $(91 / 146$, $62.3 \%)$ and stomach $(19 / 146,13.0 \%)$ were the main sites of metastasis. Both Chi-square test and logistic regression analysis showed that tumor size $(\mathrm{P}<0.05)$, tumor type $(\mathrm{P}=0.008)$ and peritumoral lymphatic vessel density (LVD) $(\mathrm{P}=0.004)$ were significantly correlated with tumor metastasis. Neither Chi-square test nor logistic regression analysis indicated that gender $(\mathrm{P}>0.05)$, age $(\mathrm{P}>0.05)$, tumor location $(\mathrm{P}>0.05)$, tumor number $(\mathrm{P}>0.05)$, chromaffin granule protein $\mathrm{A}$ [chromogranin A (CgA), P>0.05], synaptophysin (Syn, $\mathrm{P}>0.05$ ) or intratumoral LVD $(\mathrm{P}>0.05)$ had a significant correlation with tumor metastasis. Chi-square test revealed that tumor grade was significantly correlated with tumor metastasis. In conclusion, GEP-NETs may occur in multiple sites of the digestive system and lack specific clinical manifestations. Tumor size, tumor type, peritumoral LVD, total LVD and tumor grade are metastasis-related risk factors for GEP-NET patients.

\section{Introduction}

Gastroenteropancreatic neuroendocrine tumors (GEP-NETs) originate from diffuse neuroendocrine cells that are dispersed throughout the gastrointestinal tract and islets of Langerhans in the pancreas. GEP-NETs are relatively rare, accounting for $\sim 0.5 \%$ of all human cancers (1). The estimated annual incidence is $\sim 2-4.5$ cases per 100,000 with an increasing trend over recent decades, partly due to intensified awareness as well as the application of advanced technology, such as new endoscopic and imaging techniques (2-4).

These tumors, formerly named carcinoids, can involve any part of the gastrointestinal tract and the endocrine pancreas, and have the capability to synthesize and secrete neuropeptides and hormones which play a key role in causing carcinoid syndrome. The use of the term carcinoid has therefore been criticized due to its emphasis on an implied benign behavior. However, it is now clear that, despite a typically relatively indolent disease course, a large percentage of these tumors have lethal malignant potential. GEP-NETs include functional tumors, which secrete 
a variety of peptide hormones, and non-functional tumors, which may be asymptomatic and discovered by chance. Tumor extension, histological differentiation, lymph node metastasis, lymphatic invasion, and perineural invasion of the tumor are well known as the accepted features for the pathological evaluation of GEP-NETs as well as other malignant tumors of the gastrointestinal tract. Recent epidemiological studies indicate that gastric and rectal neuroendocrine neoplasms (NENs) are the most common forms of GEP-NETs, while duodenal NETs are rare accounting for only 1-2\% of all GEP-NENs (1,5). Liver and lymphatic metastases are the most common imaging findings at the time of the initial diagnosis of gastrointestinal endocrine tumors, being detectable in up to $40 \%$ of ileal and $80 \%$ of cecal lesions; furthermore, distant metastases are present in up to $60-80 \%$ of pancreatic NETs (PNETs) at first diagnosis (5).

At present, molecular markers as useful predictors of malignant behavior have been investigated in more and more studies. As neuroendocrine markers, synaptophysin (Syn) (a small vesicle-associated marker) and chromogranin A (CgA) (a large secretory granule-associated marker) are the mainstays, which may be identified histologically in patients with GEP-NETs by immunohistochemistry (IHC). In addition, the use of lymphatic vessel density (LVD) and pro-lymphangiogenic mediators as prognostic factors for tumor growth, differentiation and invasion have been reported to have a significant impact on survival in patients with GEP-NETs. In addition, the WHO proposed a grading system for GEP-NETs in 2010, which is based on the Ki-67 proliferation index (G1, $\leq 2 \%$; G2, 3-20\%; G3, $\geq 20 \%$ ) or mitotic index. The Ki-67 proliferation index was found to be strongly associated with tumor metastasis and prognosis in many studies (6).

GEP-NETs consistently have a poor prognosis. For example, $70-85 \%$ of non-functional PNETs present with unresectable disease, often with liver metastases, and their 5-year survival rate is $30-40 \%$. With the lowest survival rate of the GEP-NETs, PNETs show a median survival interval of only 24 months. Likewise, $75 \%$ of patients with small intestinal NETs either harbor liver metastases at presentation, or will develop metastases during the course of their disease. Due to the indolent disease course and poor prognosis, accurate classification and prognostication are critical for outlining the prognostic heterogeneity of this group of tumors ensuring effective treatment. There still exists controversy between pathologists and clinicians concerning the nomenclature and classification of GEP-NETs. WHO updated the classification of GEP-NETs in 2010 and all GEP-NETs were categorized as malignant tumors. In contrast, according to the International Classification of Diseases for Oncology, 3rd edition (ICD-O-3), some GEP-NETs are categorized as benign or of uncertain malignancy. Therefore, for a more accurate classification of GEP-NETs, a greater understanding of their biological behavior is required.

Timely therapeutic interventions for GEP-NET patients are needed. Neuroendocrine tumors require not only dedicated interventions to control their capacity to secrete hormones, but also, antitumor growth strategies. Somatostatin analog treatment remains a cornerstone of GEP-NET therapy (7). It has been found to be useful in controlling clinical symptoms arising from hormone secretion and slowing down disease progression. PNETs have been significantly responsive to cytotoxic chemotherapy but current prospective data are lacking (7). New treatment options for GEP-NETs have become available, and highlight the necessity of developing predictive biomarkers which will allow for appropriate and individualized selection of therapy. Early detection and surgical removal is currently the only reliable curative treatment for GEP-NET patients, especially for patients with poorly differentiated neuroendocrine carcinoma (8).

In the present research, we retrieved and analyzed information concerning clinical characteristics and metastasis-related risk factors, aiming to analyze the clinicopathological characteristics of GEP-NETs and explore the association between tumor metastasis and possible related risk factors, which can benefit early diagnosis and treatment.

\section{Materials and methods}

Participants. This study was approved and monitored by the Ethics Committee of the Second Xiangya Hospital of Central South University, China. All participants provided written informed consent. One hundred and forty-six GET-NET patients at the Second Xiangya Hospital of Central South University, China, from January 2001 to January 2015, were enrolled in this retrospective study. All of the data regarding these patients were recorded in a structured manner that included patient demographic, clinical and investigational parameters. The case notes of these patients were reviewed, and information regarding their demography, disease duration, clinical manifestations, radiological features, endoscopic features, and treatment (medical, surgical, and interventional therapy) was retrieved. According to the new WHO classification and site-specific TMN staging (9-11), patients in 0 and I stage were divided into the non-metastasis group $(n=96)$, while patients in II, III, IV stages were included in the metastasis group $(n=50)$.

We retrospected and summarized the clinical features, tumor characteristics and IHC staining results of the GEP-NETs, and collected 15 candidate risk factors for the GEP-NETs (the 15 risk factors are listed in Table I for details). The risk factors collected included patient general information, common clinical manifestations, tumor characteristics and IHC staining results. We evaluated which features act as metastasizing risk factors for GEP-NETs below.

All procedures performed in studies involving human participants were in accordance with the Ethical Standards of the Institutional and/or National Research Committee and with the 1964 Helsinki Declaration and its later amendments or comparable ethical standards.

Informed consent was obtained from all individual participants included in the study. The patients volunteered to participant in our study and they were informed in regards to the goal and content of the study. The information of all patients was kept confidential.

Tumor characteristics of the GEP-NETs. The characteristics of the tumors, such as size, number, site, type, tumor surface and infiltration depth, were assessed through the combination of CT scans, MRI and endoscopic manifestation by two experienced examiners. Tumor size, infiltration depth and presence or absence of metastatic locoregional lymph nodes were best assessed by endoscopic ultrasound (EUS). As for the patients 
Table I. Screened risk factors for GEP-NET metastasis.

\begin{tabular}{llc}
\hline Classification & \multicolumn{1}{c}{ Risk factors } & $\begin{array}{c}\text { Independent } \\
\text { variable }\end{array}$ \\
\hline General information & Gender & $\mathrm{X}_{1}$ \\
& Age $>40$ years & $\mathrm{X}_{2}$ \\
Clinical manifestation & Abdominal pain & $\mathrm{X}_{3}$ \\
& Stool changes & $\mathrm{X}_{4}$ \\
Common tumor & Blood in stool & $\mathrm{X}_{5}$ \\
characteristics & Tumor size & $\mathrm{X}_{6}$ \\
& Tumor no. & $\mathrm{X}_{7}$ \\
& Tumor site & $\mathrm{X}_{8}$ \\
Pathological IHC & Tumor type & $\mathrm{X}_{9}$ \\
parameters & Infiltration depth & $\mathrm{X}_{10}$ \\
& CgA & $\mathrm{X}_{11}$ \\
& Syn & $\mathrm{X}_{12}$ \\
& Peritumoral LVD & $\mathrm{X}_{13}$ \\
& Intratumoral LVD & $\mathrm{X}_{14}$ \\
& Total LVD & $\mathrm{X}_{15}$ \\
\hline
\end{tabular}

GEP-NET, gastroenteropancreatic neuroendocrine tumor; CgA, chromogranin A; Syn, synaptophysin; IHC, immunohistochemistry; LVD, lymphatic vessel density.

who underwent surgical resection, the characteristics of the tumors were assessed directly through gross morphology by two pathologists.

Immunohistochemistry. Sections for immunohistochemistry (IHC) were stained using the avidin-biotin complex (ABC) method. Four-micrometer thick sections were cut from the blocks, deparaffinized with xylene and dehydrated through graded concentrations of alcohol. Endogenous peroxidase activity was blocked with $3 \%$ hydrogen peroxide in methanol for $10 \mathrm{~min}$. After antigen retrieval, the sections were then incubated with primary antibodies podoplanin (1:200 dilution, sc-59347; Santa Cruz Biotechnology, Inc., Santa Cruz, CA, USA), CgA (1:50, MAB-0202, Maxim) or Syn (1:50, RM-9111; Huaruikang Biotech Science Co., Ltd, Wuhan, China) or Ki-67 (1:100, ab15580; Abcam, Cambridge, UK) overnight at $4^{\circ} \mathrm{C}$, with appropriate negative and positive controls. Then the sections were incubated with the secondary antibody (Universal HRP Multimer) for $8 \mathrm{~min}$ at $37^{\circ} \mathrm{C}$. Subsequently, the slides were treated with the DAB $+\mathrm{H}_{2} \mathrm{O}_{2}$ substrate for 8 min followed by counterstaining with hematoxylin and the bluing reagent at $37^{\circ} \mathrm{C}$. The reaction buffer (pH 7.6, Tris buffer) was used as a wash solution.

Semi-quantitative methods were used to describe the dyeing conditions of CgA or Syn positively stained tumor cells according to the percentage and degree of staining of positive cells. The extent of positive staining was semi-quantitatively assessed as: $0,<5 \%$ staining; $1+, 5-25 \%$ staining; $2+, 26-50 \%$ staining; $3+, 51-75 \%$ staining; and $4+, \geq 75 \%$ staining. The Ki-67 proliferation index was determined by assessing the percentage of positively staining tumor cell

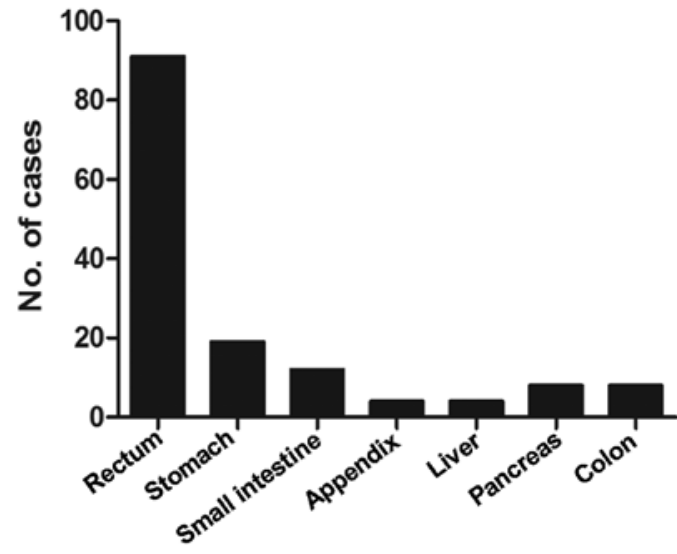

Figure 1. Distribution of the 146 patients diagnosed pathologically with GEP-NETs from January 2001 to January 2015 at the Second Xiangya Hospital of Central South University according to the organ system. GEP-NETs, gastroenteropancreatic neuroendocrine tumors.

nuclei in 2,000 cells in areas with the highest degree of nuclear labelling where possible. All slides were evaluated the same day by two pathologists to minimize the variability of the results.

LVD. LVD was assessed with digital image analysis, applying podoplanin as a marker for lymphatic vessel endothelium (12). Three most intensively vascularized intratumoral fields were acquired. In each field, vessels were marked manually and then counted automatically. Eventually, LVD was calculated as the number of vessels in the most vascularized field. A single immunoreactive endothelial cell or a cluster of endothelial cells (brown in case of a lymphatic vessel) separated from other vessels, was counted as a single vessel. Vessels with and without lumen were counted.

Statistical analysis and the establishment of the prediction equation. Clinical and pathological parameters including IHC staining (measured semi-quantitatively) were analyzed using the Chi-square test and Student's t-test. Logistic regression analysis and Chi-square test were both used to conduct multivariate analyses of metastasis-related risk factors of GEP-NETs. Statistical significance was accepted at $\mathrm{P}<0.05$. All analyses were performed using SPSS version 17.0. Prediction equation: $\ln [\mathrm{P} /(1-\mathrm{P})]=\mathrm{e}+\beta_{1} \mathrm{X}_{1}+\beta_{2} \mathrm{X}_{2}+\beta_{3} \mathrm{X}_{3}+$ $\beta_{4} X_{4}+\beta_{5} X_{5}+\beta_{6} X_{6}+\beta_{7} X_{7}+\beta_{8} X_{8}+\beta_{9} X_{9}+\beta_{10} X_{10}+\beta_{11} X_{11}+$ $\beta_{12} X_{12}+\beta_{13} X_{13}+\beta_{14} X_{14}+\beta_{15} X_{15}$. We used a backward method to analyze all 15 variables. The final equation was confirmed until no more variables could be deleted from the current model. We also estimated odds ratio for each variable and their $95 \%$ confidence interval.

\section{Results}

Common characteristics of the GEP-NETs. In our retrospective study, the total study population included 96 males and 50 females with a mean age of $49.26 \pm 13.31$ years. The composition of the cases enrolled in our study is described in Fig. 1 according to tumor location. The most common underlying tumor sites were the rectum $(62.3 \%)$, stomach $(13.0 \%)$, and small intestine $(8.2 \%)$. Abdominal pain $(n=88)$, character 
Table II. Univariate analysis of risk factors for metastasis of GEP-NETs based on clinical manifestation parameters.

\begin{tabular}{lrccc}
\hline Risk factors & No. & $\begin{array}{c}\text { Non-metastasis } \\
\text { group }\end{array}$ & $\begin{array}{c}\text { Metastasis } \\
\text { group }\end{array}$ & P-value \\
\hline Gender & & & 37 & $>0.05$ \\
$\quad$ Male & 96 & 59 & 13 & \\
Female & 50 & 37 & & $>0.05$ \\
Age (years) & & & 46 & \\
$\geq 40$ & 112 & 66 & 4 & \\
$<40$ & 34 & 30 & 34 & $>0.05$ \\
Abdominal pain & & & 16 & \\
Yes & 88 & 54 & & $<0.05$ \\
No & 58 & 42 & 30 & \\
Stool change & & & 20 & \\
Yes & 58 & 28 & & $<0.05$ \\
No & 88 & 68 & 25 & \\
Melaena & & & 25 & \\
Yes & 33 & 8 & & \\
No & 113 & 88 & & \\
\hline
\end{tabular}

GEP-NETs, gastroenteropancreatic neuroendocrine tumors.

Table III. Univariate analysis of risk factors for metastasis of GEP-NETs based on common tumor characteristics.

\begin{tabular}{|c|c|c|c|c|}
\hline Risk factors & No. & $\begin{array}{l}\text { Non-metastasis } \\
\text { group }\end{array}$ & $\begin{array}{c}\text { Metastasis } \\
\text { group }\end{array}$ & P-value \\
\hline Tumor size (cm) & & & & $<0.05$ \\
\hline$<1$ & 72 & 62 & 10 & \\
\hline $1-2$ & 44 & 24 & 20 & \\
\hline$>2$ & 30 & 8 & 22 & \\
\hline No. of tumors & & & & $>0.05$ \\
\hline 1 & 120 & 75 & 45 & \\
\hline$\geq 2$ & 26 & 21 & 5 & \\
\hline Tumor type & & & & $<0.05$ \\
\hline Ulcerative & 28 & 10 & 18 & \\
\hline Non-ulcerative & 42 & 32 & 10 & \\
\hline Tumor site & & & & $>0.05$ \\
\hline Stomach & 19 & 10 & 9 & \\
\hline Small intestine & 12 & 6 & 6 & \\
\hline Appendix & 4 & 3 & 1 & \\
\hline Liver & 4 & 3 & 1 & \\
\hline Pancreas & 8 & 1 & 7 & \\
\hline Colon & 8 & 3 & 5 & \\
\hline Rectum & 91 & 70 & 21 & \\
\hline Infiltration depth & & & & $>0.05$ \\
\hline Infiltration & 13 & 7 & 6 & \\
\hline Non-infiltration & 31 & 20 & 11 & \\
\hline
\end{tabular}

Once the lesion involved the submucosa it was defined as tumor infiltration by endoscopic ultrasound examination. GEP-NETs, gastroenteropancreatic neuroendocrine tumors.
Table IV. Correlation between the expression of CgA and Syn and GEP-NETs.

\begin{tabular}{lrrrr}
\hline & $\mathrm{N}$ & $\mathrm{n}$ & $\chi^{2}$ & P-value \\
\hline CgA & & & 1.635 & $>0.05$ \\
Positive & 77 & 30 & & \\
Negative & 39 & 8 & & \\
Syn & & & 0.001 & $>0.05$ \\
$\quad$ Positive & 118 & 41 & & \\
Negative & 6 & 2 & & \\
\hline
\end{tabular}

CgA, chromogranin A; Syn, synaptophysin; GEP-NETs, gastroenteropancreatic neuroendocrine tumors; $\mathrm{N}$, number of positive cases; $\mathrm{n}$, number of cases with metastasis.

change of stool $(n=58)$ and melaena $(n=30)$ were the most common non-specific clinical symptoms.

Clinical manifestation parameters indicated for the metastasis of GEP-NETs. General information of the patients, such as age and gender, and the three most common non-specific clinical symptoms including abdominal pain, character change of stool and melaena, were collected in our study. All the five candidate variables evaluated in the retrospective cohort were analyzed by Chi-square test (Table II). According to the statistical results, tumor metastasis was more likely to occur in the GEP-NET patients accompanied by change of stool and melaena. Age, gender and abdominal pain were not determined to be metastasis risk factors of GEP-NETs.

Ulcer formation and a larger size of the lesion, not the infiltration depth of the tumor, participate in higher metastasis risk of GEP-NETs. Tumor size, number, type, site and infiltration depth were important parameters to evaluate the characteristics and progression of tumors. Among the five candidate variables from the common tumor characteristics evaluated in the retrospective cohort, two were associated with GEP-NETs by Chi-square test and matching Chi-square test (Table III): i) tumor type: ulcerative type of neuroendocrine tumor metastasizes more likely than the non-ulcer ones; and ii) tumor size: the larger the tumor, the higher the risk to transfer. The detailed information such as tumor number, size, surface obtained by endoscopy is documented in Fig. 2.

When assessing the role of infiltration depth achieved by endoscopic ultrasonography in tumor metastasis, there was no statistical significance between the metastatic group and the non-metastatic one. Performance under endoscopic ultrasonography is shown in Fig. 3. The pathological biopsy is the gold standard when it comes to determining tumor metastases and endoscopy plays an important role in tissue biopsy and endoscopic therapy.

$C g A$ and Syn are not indicators for the metastasis of GEP-NETs. CgA and Syn are considered as the most important biomarkers for the pathological IHC index in diagnosing neuroendocrine tumors. In the sections for IHC, $\mathrm{CgA}$ and Syn were both sensitive and specific biomarkers for the 

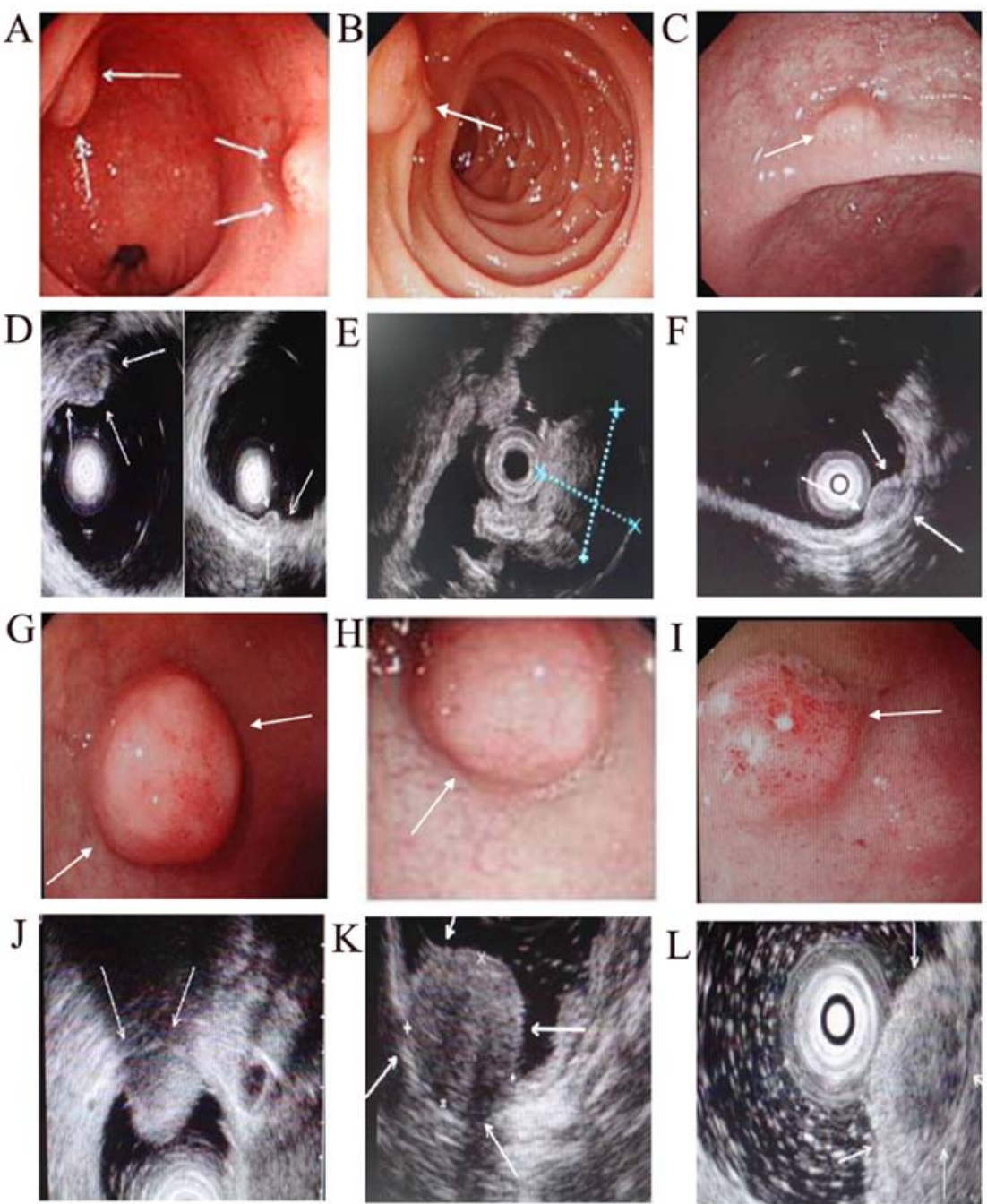

Figure 2. Characteristics of the tumors under gastrointestinal endoscopy and EUS. (A and D) Two neuroendocrine carcinomas with wide-base hemispherical uplift were identified by gastrointestinal endoscopy and EUS, which were located in the submucous and mucous layer of the rectum. (B and E) A single rectal neuroendocrine carcinoma, located in the submucous layer, infiltrating the muscularis propria was confirmed by pathological biopsy examination. ( $\mathrm{C}$ and F) A rectal neuroendocrine carcinoma with a size $\sim 0.4 \mathrm{x} 0.7 \mathrm{~cm}$, located in the limit of the mucous layer was confirmed by EUS. (G and J) A duodenal neuroendocrine carcinoma with a size $\sim 1.98 \times 0.95 \mathrm{~cm}$, originating from the mucous layer was confirmed by EUS. However, pathological biopsy examination showed that the lesion had infiltrated the propria muscularis layer. ( $\mathrm{H}$ and $\mathrm{K}$ ) A non-ulcerative neuroendocrine carcinoma in the anterior duodenum wall. EUS found a low level echo located in the submucous layer with a size $\sim 1.37 \mathrm{x} 1.55 \mathrm{~cm}$ and clear edge. (I and L) An ulcerative neuroendocrine carcinoma in the anterior duodenum wall, a tumor with a size $\sim 1.0 \times 1.2 \mathrm{~cm}$ and a congestive and rough surface was found located in the muscularis propria. Lifting sign was not obvious. EUS, endoscopic ultrasound.

presence of GEP-NETs (Fig. 3), whereas they act more like diagnostic biomarkers than risk factors for metastasis. The study showed no statistically significant impact on metastasis for CgA-positive (77/116) or Syn-positive (118/124) compared with CgA-negative (39/116) or Syn-negative (6/124) samples by Chi-square test (Table IV).

Higher Ki-67 proliferation index participates in higher risk of metastasis of GEP-NETs. The Ki-67 proliferation index is an important and independent prognostic marker in GEP-NETs (Fig. 3). The prognosis of GEP-NETs is closely related to the metastasis of the disease. The test indicated that the Ki-67 proliferation index was significantly correlated with tumor metastasis. Overall, 70 patients $(66.67 \%)$ had G1 tumors and G2 and G3 tumors were seen in 28 (26.67\%) and 7 (6.67\%) cases, respectively. When stratified according to grade, metastasized tumors were noted in $14.29 \%(60 / 70)$ of the G1
Table V. Relationship between tumor grade and metastasis of GEP-NETs.

\begin{tabular}{lcccc}
\hline Grade & No. & $\begin{array}{c}\text { Non-metastasis } \\
\text { group }\end{array}$ & $\begin{array}{c}\text { Metastasis } \\
\text { group }\end{array}$ & P-value \\
\hline G1 & 70 & 60 & 10 & $<0.05$ \\
G2 & 28 & 4 & 24 & \\
G3 & 7 & 2 & 5 & \\
\hline
\end{tabular}

GEP-NETs, gastroenteropancreatic neuroendocrine tumors.

cases, $85.71 \%(24 / 28)$ of the G2 cases, and $71.43 \%(5 / 7)$ of the G3 cases. The metastasis of the disease was correlated with increased $\mathrm{Ki}-67$ values $(\mathrm{P}<0.05)$ by Chi-square test (Table V). 


\section{A}
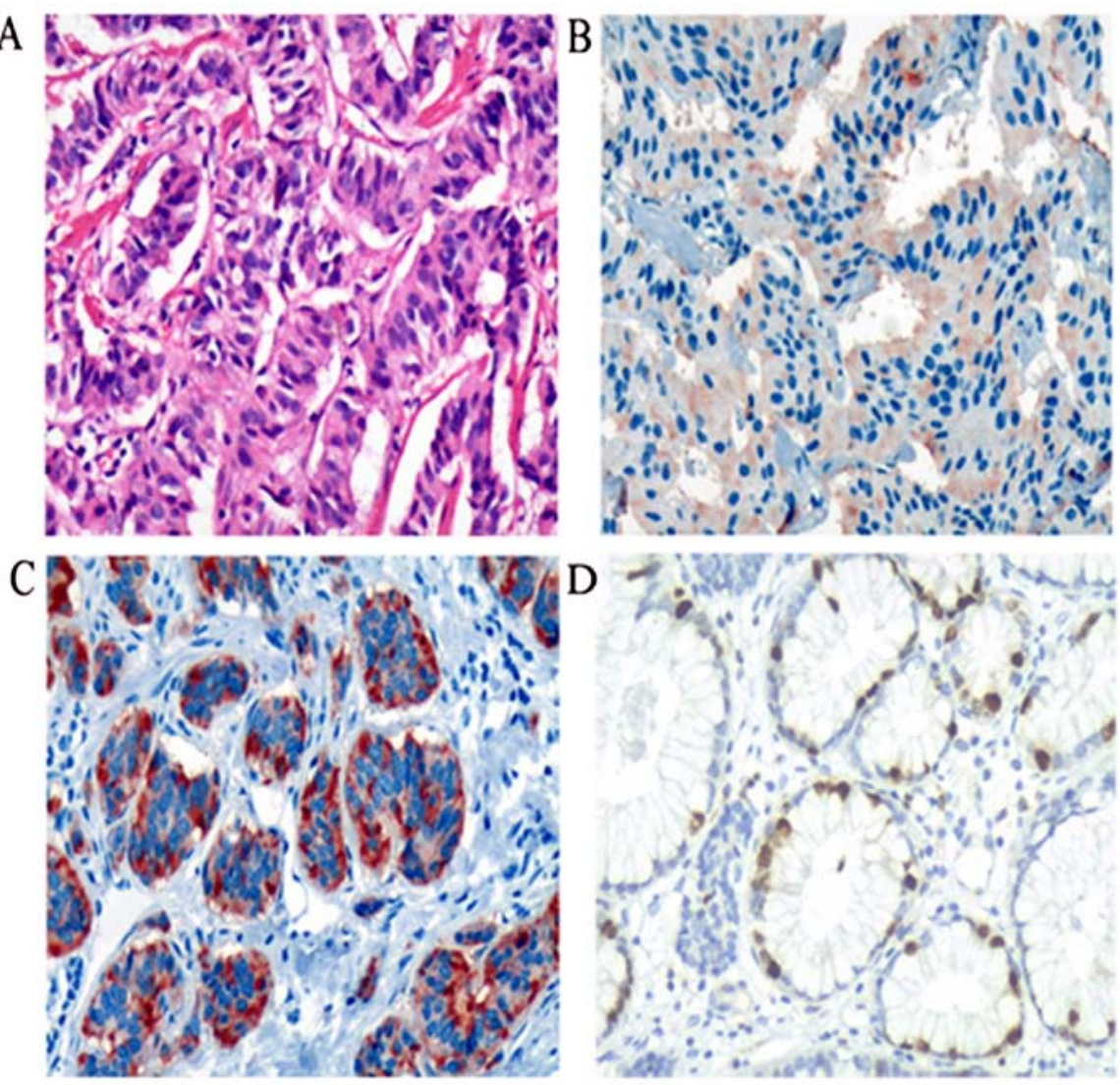

Figure 3. Expression levels of CgA and Syn are positive in the presence of GEP-NETs. (A) Panoramic view of non-metastatic rectal neuroendocrine carcinoma under H\&E staining (magnification, x200). Moderate expression of (B) CgA and (C) Syn immunostaining of non-metastatic rectal neuroendocrine carcinoma (magnification, x200). (D) Moderate expression of Ki-67 in moderately differentiated NETs (G2, 5\%) (magnification, x200). CgA, chromogranin A; Syn, synaptophysin; GEP-NETs, gastroenteropancreatic neuroendocrine tumors.

Table VI. Relationship between LVD and metastasis of GEP-NETs.

\begin{tabular}{lcccc}
\hline & No. & LVD & $\mathrm{t}$ & P-value \\
\hline $\begin{array}{l}\text { Lymph node } \\
\text { involvement }\end{array}$ & & & -2.373 & 0.021 \\
$\quad$ Positive & 14 & $11.83 \pm 2.16$ & & \\
$\quad$ Negative & 47 & $10.65 \pm 1.31$ & & \\
$\quad \begin{array}{l}\text { Peritumoral LVD } \\
\text { Without metastasis }\end{array}$ & 28 & $6.23 \pm 1.18$ & & \\
$\quad$ Metastasis & 33 & $7.33 \pm 1.51$ & & \\
$\quad$ Intratumoral LVD & & & 1.655 & 0.104 \\
$\quad$ Without metastasis & 28 & $4.23 \pm 0.59$ & & \\
$\quad$ Metastasis & 33 & $4.00 \pm 0.45$ & & \\
Total LVD & & & -1.949 & 0.056 \\
$\quad$ Without metastasis & 28 & $10.46 \pm 1.21$ & & \\
$\quad$ Metastasis & 33 & $11.27 \pm 1.78$ & & \\
\hline
\end{tabular}

LVD, lymphatic vessel density; GEP-NETs, gastroenteropancreatic neuroendocrine tumors.

High tumor LVD is associated with a higher risk of metastasis of GEP-NETs. LVD using podoplanin as a marker for
Table VII. Risk factors associated with GEP-NETs according to logistic regression analysis.

\begin{tabular}{lrrr}
\hline Risk factors & B & P-value & Odds ratio \\
\hline Tumor size & 2.277 & 0.037 & 9.752 \\
Total LVD & -3.061 & 0.036 & 0.047 \\
Peritumoral LVD & 4.129 & 0.013 & 62.104 \\
Tumor type & -3.092 & 0.033 & 0.045 \\
Blood in stool & 3.333 & 0.089 & 28.020 \\
Constant & 7.283 & 0.275 & $1,455.043$ \\
\hline
\end{tabular}

GEP-NETs, gastroenteropancreatic neuroendocrine tumors; B, independent variable index; LVD, lymphatic vessel density.

lymphatic vessel endothelium, which is often expressed at the leading invasive edge of tumors, has been implicated in tumor progression. Podoplanin immunonegativities and immunopositivities are presented in Fig. 3. In our study, the expression of podoplanin with brown staining was found in lymphatic epithelial cells or surrounding tumor sites.

According to the test, there was statistical significance between the lymph node metastasis group and without lymph node metastasis group $(\mathrm{P}<0.05)$ (Table VI). However, when analyzing the roles of peritumoral and intratumoral LVD 

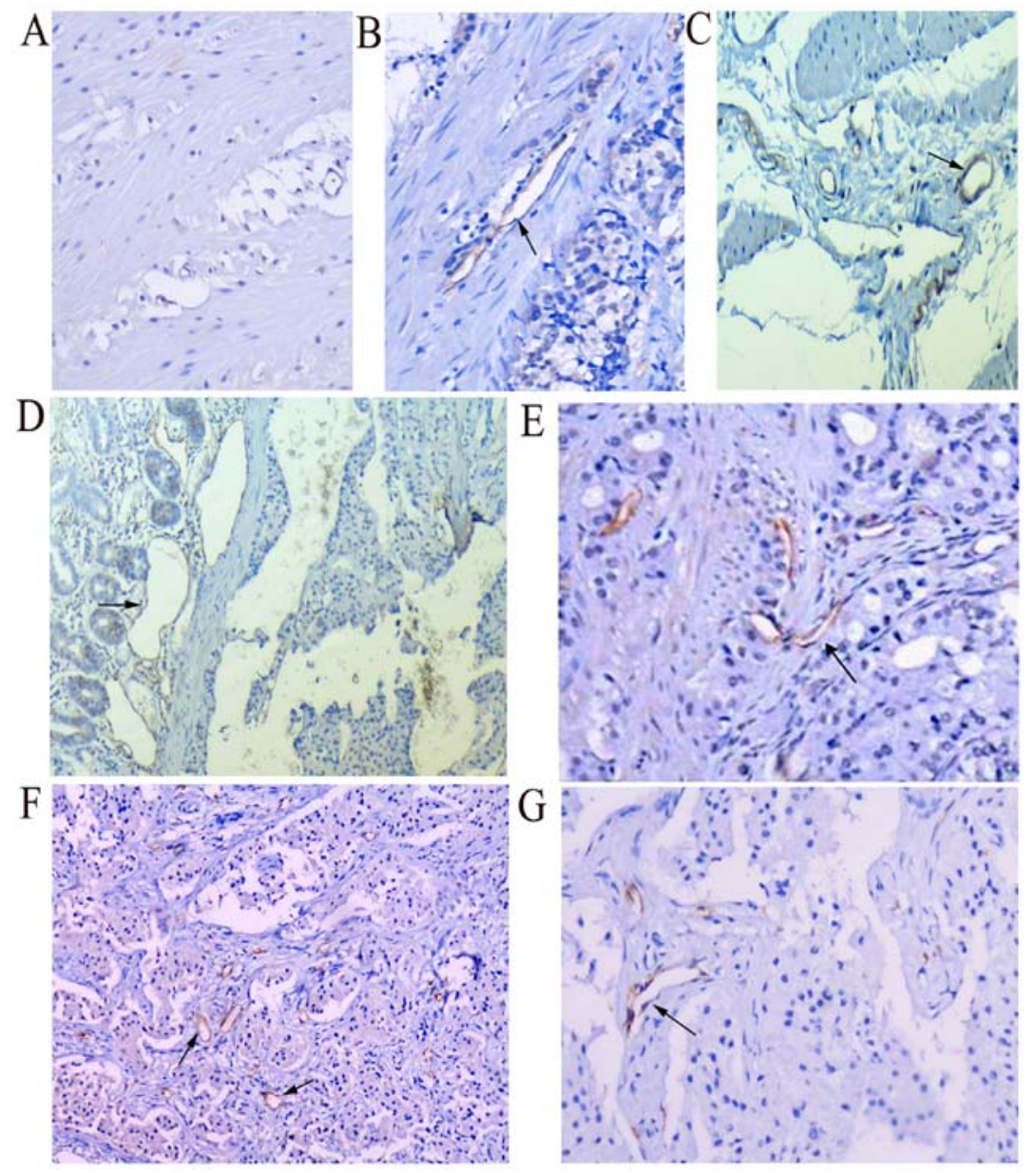

Figure 4. Total LVD is a related risk factor for metastasis. (A) No obvious positive expression of podoplanin was noted in the normal rectal tissues (magnification, x100). (B) Moderate positive expression of podoplanin was noted in non-metastatic rectal GEP-NETs, with endothelium of lymph vessels staining brown (magnification, x100). (C) Obvious positive expression was noted in metastatic rectal GEP-NETs, with increased endothelium of lymph vessels staining brown. LVD was greater than that in the non-metastatic ones (magnification, $x 100)$. (D and E) Positive expression of podoplanin was noted in endothelium of lymphatic vessels in non-metastatic duodenal neuroendocrine carcinoma; (D) peritumoral lymphatic vessels appeared to be dilated obviously (magnification, $\mathrm{x} 200$ ); however (E) intratumoral lymphatic vessels were flat and atretic (magnification, $\mathrm{x} 200$ ). (F) More round and dilated peritumoral lymphatic vessels were noted in duodenal GEP-NETs with liver invasion (magnification, x200), however (G) intratumoral lymphatic vessels were flat and atretic; not significant in number (magnification, x200). LVD, lymphatic vessel density; GEP-NETs, gastroenteropancreatic neuroendocrine tumors.

in the tumor metastasis, we found only peritumoral, not intratumoral LVD, to be significantly associated with tumor metastasis (Fig. 4).

Metastasis-related variables associated with GEP-NETs according to logistic regression analysis. All the dependent variables in the table used logistic regression to explore the association between tumor metastasis and possible related risk factors. In our study, logistic regression analysis using backward attribute selection methods indicated that tumor size, tumor type, total LVD, peritumoral LVD, melaena were significant and independent risk factors (Table VII). Logistic regression equation: $\ln \left[(\mathrm{P} /(1-\mathrm{P})]=7.283+2.277 \mathrm{X}_{6}-3.092 \mathrm{X}_{9}\right.$ $+4.129 \mathrm{X}_{13}-3.061 \mathrm{X}_{15}$.

\section{Discussion}

GEP-NETs are rare tumors, characterized by heterogeneous biological behavior and clinical course. The Surveillance, Epidemiology, and End Results (SEER) database suggests that their prevalence has increased dramatically over the last three decades, due to an increase in the actual number of cases and/or more effective detection of this disease $(13,14)$. The prognosis of GEP-NETs mainly depends on whether the tumors metastasize or not. Therefore, early detection and evaluation of metastasis risk have been currently the most urgent task for GEP-NET patients. To our best of our knowledge, among the 15 candidate variables in our retrospective cohort, four were associated with GEP-NETs by Chi-square test and logistic regression: i) tumor type, ii) tumor size, iii) peritumoral LVD, and iv) total LVD.

The clinical presentations of patients with GEP-NETs depend on the hormonal activity of the tumors and on their location and extent. Numerous tumors produce low levels of substances that are clinically insignificant or secrete metabolically inactive or inappropriately processed substances. Most GEP-NETs are non-functional and present fairly late with mass effects, distant metastasis, or both. Frequently, symptoms are vague and unspecific. In our study, we found that the most common clinical manifestations of non-functional GEP-NETs were abdominal pain $(n=88)$, character change of stool $(n=58)$ and melaena $(n=33)$, however, there 
was no statistical significance between the symptoms above and metastasis. Kuiper et al found that common symptoms of non-functional neuroendocrine tumors were abdominal pain, weight loss, anorexia, jaundice, nausea and vomiting and intra-abdominal haemorrhage (15). None of the common symptoms were reported to be specific to GEP-NETs. The classic syndromes associated with functioning GEP-NETs include the carcinoid syndrome, which is the result of the interaction of tumor factors such as 5-hydroxytryptamine (5-HT) (serotonin), kinins, and kallikrein entering the systemic circulation, leading to flush, diarrhea, and other features of carcinoid syndrome. Occasionally, carcinoid crisis, which is an overwhelming release of bioactive amines, can develop in patients with foregut and midgut carcinoids, and can present with hypotension (rarely hypertension), arrhythmias, wheezing, and delirium. Recurrent hypoglycemia is a typical symptom of insulinomas. These tumors manifest themselves in adrenergic symptoms such as tachycardia, anxiety, sweating, and palpitations, even loss of consciousness as a symptom. Recurrent duodenal ulcer and gastroesophageal reflux are the primary symptoms of duodenal or pancreatic gastrinoma.

Tumor characteristics,such as location, size, type, number, infiltration depth, and the presence of metastases, were assessed by CT scan, MRI and endoscopic procedures; the latter one has been recommended for GI tract GEP-NENs, and plays a pivotal role in the diagnostic work-up and the therapy of GEP-NENs. In our study, tumor type and size were found to correlate significantly with the metastasis of tumors. Ulcerative types are more likely to transfer than the non-ulcerative ones. In addition, a size $>2 \mathrm{~cm}$ acts as a high risk factor which is consistent with what has been reported in other research (16). Schott et al reported that more than $80 \%$ of cases are $<2 \mathrm{~cm}$ and are benign $(17,18)$. All other tumors, such as gastrinomas, glucagonomas, and VIPomas, and especially the numerous non-functional NETs of the pancreas, are usually $>2 \mathrm{~cm}$ and are malignant. In a Japanese report, metastasis showed a significant correlation with tumor size. Tumors $<1 \mathrm{~cm}$ and confined to the submucosa without vessel invasion did not show any metastasis. According to the latest WHO classification and site-specific TMN staging, the dimension recommended as a metastasis risk factor is different owing to the different site. For the stomach, appendix, colon and rectum tumors, lower size limits were defined based on current information on the biology of tumors. The size limits indicated for T1 $(<1 \mathrm{~cm})$ are those defined by the WHO for tumors with 'benign behavior'. Deeply invasive tumors are included over T2 $(1-2 \mathrm{~cm})$ according to site-specific clinicopathological correlations $(19,20)$. Unfortunately more and more cases of small rectal carcinoids (even $<5 \mathrm{~mm}$ ) accompanied by multiple liver metastasis have been reported in the literature $(21,22)$. As a rule, tumors confined to the pancreas, $<2 \mathrm{~cm}$ in size, show a benign behavior. However in the pancreas the size limit given for T2 $(2-4 \mathrm{~cm})$ needs to be validated.

It is worth noting that location and infiltration depth are considered to be high risk factors in other studies. The relation between location, infiltration depth and metastasis warrants more in-depth research. During the last decade, the development of new and more sophisticated diagnostic and therapeutic endoscopic instruments and tools have enriched the armamentarium available to the endoscopist. GEP-NENs, however, still represent a clinical challenge to the endoscopist because of their small size, which may render their search very difficult. At present, gastric, duodenal, and rectal NENs are diagnosed with increased frequency due to the widespread use of diagnostic upper and lower endoscopic examinations (23). Tumor size, depth of infiltration within the GI wall, and presence or absence of metastatic locoregional lymph nodes are important parameters, and can be detected by endoscopy, especially EUS. EUS has a crucial role in the search for GEP-NENs of the GI wall, since it provides information on the size, depth of invasion and locoregional metastasis. Also, EUS-guided fine-needle aspiration can also provide a definite diagnosis and useful information for the correct management of this type of lesion. For many years EUS has been advocated as the best available technique for imaging the pancreas and the extrahepatic biliary tree $(10,24)$. High resolution images of the main pancreatic duct and surrounding parenchyma can be achieved and structures as small as $2-3 \mathrm{~mm}$ can be distinguished thanks to the small distance between the transducer and the gland. EUS can detect $45-60 \%$ of duodenal lesions and $90-100 \%$ of pancreatic lesions.

Pathological examination of biopsies or surgical specimens reveals the verification of the neuroendocrine nature of the tumor by IHC, for markers such as keratin, CgA, Syn, neuron-specific enolase (NSE), grimelius, Ki67 and CD56, which provides a promising new diagnostic method for NETs. Although research into specific biomarkers to detect GEP-NETs is ongoing, all the above-mentioned studies are non-conclusive, and further research and validation studies are needed before these diagnostic tools can be used in practice. In clinical study, CgA and Syn are widely used in the diagnosis of neuroendocrine tumors. Although non-conclusive, they have received the validation of many researchers for their high sensitivity and specificity (25). We also speculate concerning the role they play in tumor metastasis. In our study, we found that IHC of biopsy specimens using a selected panel of markers, including $\mathrm{CgA}$ and Syn could be used to help diagnose NETs with high sensitivity and specificity. Our positivity rate of $66.4 \%$ for $\mathrm{CgA}$ in GEP-NETs is similar to the results of a recent study (26). In contrast, the positivity rate of Syn was $95.2 \%$ in our study, again similar to the results of previous studies $(27,28)$, confirming that Syn is superior to $\mathrm{CgA}$ as an IHC marker. But both markers have no significant relationship with the metastasis of NETs. We recommend CgA and Syn as highly specific and sensitive neuroendocrine markers in the diagnosis of NETs, but not markers for metastasis.

$\mathrm{Ki}-67$ is an important marker of cell proliferation which is active in the cell cycle phases G1, S and G2 and during mitosis. A high Ki-67 proliferation index indicates abnormal proliferation and the aggressiveness of a tumor. Many retrospective studies have demonstrated that Ki-67 shows a good correlation with tumor size, vessel and the behavior of neuroendocrine tumors. Richards-Taylor et al provided a substantial body of evidence related to the use of Ki-67 as a prognostic marker in GEP-NETs (6). Özaslan et al reported that the grade and stage of the disease increased in line with a higher Ki-67 index (29). In our study, the Ki-67 proliferation index (tumor grade) was significantly correlated with the metastasis of GEP-NETs. 
Lymph node metastasis is a common occurrence in GEP-NETs. Malignant cells spread from their primary site to regional lymph nodes via the lymphatics at an early stage in the dissemination of the tumors (30). Recent studies have indicated that tumor lymphangiogenesis, the growth of new lymphatic vessels, is linked to the formation of lymph node metastases when observing the LVD and the overexpression of podoplanin (31-33). Our data used LVD as a marker of lymphangiogenesis which was obtained or calculated from the expression of PDPN. As we know, PDPN is a $38-\mathrm{kDa}$ mucin-type transmembrane glycoprotein with extensive $O$-glycosylation and high sialic acid content, and has been implicated in tumor progression. PDPN is expressed in lymphatic endothelial cells as well as cancer cells. The mechanism underlying the impact of lymphangiogenesis or LVD on tumor progression remains uncertain. Previous studies have demonstrated a link between tumor-induced lymphangiogenesis and enhanced tumor metastasis to sentinel lymph nodes and remote metastasis based on the evidence that the intratumoral vessels are newly proliferating and not trapped pre-existing or hyperplastic lymph vessels. The main significance of proliferating intratumoral lymph vessels is that they could provide a possible route for the spread of tumors to local lymph nodes. However, whether the effects of lymphangiogenesis on the risk of metastasis are due to increased lymphatic permeability or increased abundance of intratumoral and/or peritumoral lymphatics remains controversial. Our study showed that total LVD was a related risk factor for metastasis, and it was noted that peritumoral, not intratumoral, LVD acted as a statistically significant factor related to metastasis. We speculate that the invasion of increased peritumoral LVD may play an important role in tumor metastasis, similar to the results of Pastushenko et al (34). Understanding the mechanism of peritumoral lymphatic prolification and growth may benefit the diagnosis and treatment of GEP-NETs.

In conclusion, the incidence of GEP-NET has shown a marked increase during the last decade. Due to its indolent disease courses and poor prognosis, early detection and timely assessment of the risks of metastasis remain critical issues. We found that tumor size, tumor type, Ki-67 proliferation index, peritumoral LVD and total LVD may be metastasis-related risk factors for GEP-NET patients. In addition, endoscope and neuroendocrine markers, such as $\mathrm{CgA}$ and Syn, play important roles in identifying the characteristics of tumors and aid in the diagnosis and treatment of GEP-NETs.

\section{Acknowledgements}

This study was supported by the National Natural Science Foundation of China (no. 81172299) and the Research Program from the Science and Technology Department of Hunan Province, China (S2012F1023).

\section{References}

1. Meeker A and Heaphy C: Gastroenteropancreatic endocrine tumors. Mol Cell Endocrinol 386: 101-120, 2014.

2. Pinchot SN, Holen K, Sippel RS and Chen H: Carcinoid tumors. Oncologist 13: 1255-1269, 2008.

3. Strosberg JR, Nasir A, Hodul P and Kvols L: Biology and treatment of metastatic gastrointestinal neuroendocrine tumors. Gastrointest Cancer Res 2: 113-125, 2008.
4. Ramage JK, Ahmed A, Ardill J, Bax N, Breen DJ, Caplin ME, Corrie P, Davar J, Davies AH, Lewington V, et al; UK and Ireland Neuroendocrine Tumour Society: Guidelines for the management of gastroenteropancreatic neuroendocrine (including carcinoid) tumours (NETs). Gut 61: 6-32, 2012

5. Klöppel G, Rindi G, Anlauf M, Perren A and Komminoth P: Site-specific biology and pathology of gastroenteropancreatic neuroendocrine tumors. Virchows Arch 451 (Suppl 1): S9-S27, 2007.

6. Richards-Taylor S, Ewings SM, Jaynes E, Tilley C, Ellis SG, Armstrong T, Pearce $\mathrm{N}$ and Cave J: The assessment of Ki-67 as a prognostic marker in neuroendocrine tumours: A systematic review and meta-analysis. J Clin Pathol 69: 612-618, 2016.

7. Baudin E, Planchard D, Scoazec JY, Guigay J, Dromain C, Hadoux J, Debaere T, Elias D and Ducreux M: Intervention in gastro-enteropancreatic neuroendocrine tumours. Best Pract Res Clin Gastroenterol 26: 855-865, 2012.

8. Knigge U and Hansen CP: Surgery for GEP-NETs. Best Pract Res Clin Gastroenterol 26: 819-831, 2012.

9. Li ZS and Li Q: The latest 2010 WHO classification of tumors of digestive system. Zhonghua Bing Li Xue Za Zhi 40: 351-354, 2011 (In Chinese).

10. Yang Z, Tang LH and Klimstra DS: Gastroenteropancreatic neuroendocrine neoplasms: Historical context and current issues. Semin Diagn Pathol 30: 186-196, 2013.

11. Capelli P, Fassan M and Scarpa A: Pathology - grading and staging of GEP-NETs. Best Pract Res Clin Gastroenterol 26: 705-717, 2012.

12. Raica M, Cimpean AM and Ribatti D: The role of podoplanin in tumor progression and metastasis. Anticancer Res 28: 2997-3006, 2008.

13. Gastrointestinal Pathology Study Group of Korean Society of Pathologists; Cho MY, Kim JM, Sohn JH, Kim MJ, Kim KM, Kim WH, Kim H, Kook MC, Park DY, Lee JH, et al: Current trends of the incidence and pathological diagnosis of gastroenteropancreatic neuroendocrine tumors (GEP-NETs) in Korea 2000-2009: Multicenter study. Cancer Res Treat 44: 157-165, 2012.

14. Fraenkel M, Kim MK, Faggiano A and Valk GD: Epidemiology of gastroenteropancreatic neuroendocrine tumours. Best Pract Res Clin Gastroenterol 26: 691-703, 2012.

15. Kuiper P, Verspaget HW, Overbeek LI, Biemond I and Lamers CB: An overview of the current diagnosis and recent developments in neuroendocrine tumours of the gastroenteropancreatic tract: The diagnostic approach. Neth J Med 69: 14-20, 2011.

16. Solcia E, Rindi G, Paolotti D, La Rosa S, Capella C and Fiocca R: Clinicopathological profile as a basis for classification of the endocrine tumours of the gastroenteropancreatic tract. Ann Oncol 10 (Suppl 2): S9-S15, 1999.

17. Schott M, Klöppel G, Raffel A, Saleh A, Knoefel WT and Scherbaum WA: Neuroendocrine neoplasms of the gastrointestinal tract. Dtsch Arztebl Int 108: 305-312, 2011.

18. Zhou X, Xie H, Xie L, Li J, Cao W and Fu W: Endoscopic resection therapies for rectal neuroendocrine tumors: A systematic review and meta-analysis. J Gastroenterol Hepatol 29: 259-268, 2014.

19. Rindi G, Klöppel G, Alhman H, Caplin M, Couvelard A, de Herder WW, Erikssson B, Falchetti A, Falconi M, Komminoth P, et al: TNM staging of foregut (neuro)endocrine tumors: A consensus proposal including a grading system. Virchows Arch 449: 395-401, 2006.

20. Rindi G, Klöppel G, Couvelard A, Komminoth P, Körner M, Lopes JM, McNicol A-M, Nilsson O, Perren A, Scarpa A, et al: TNM staging of midgut and hindgut (neuro) endocrine tumors: A consensus proposal including a grading system. Virchows Arch 451: 757-762, 2007.

21. Tsuboi K, Shimura T, Suzuki H, Mochiki E, Haga N, Masuda N, Soda M, Yamamoto H, Asao T and Kuwano H: Liver metastases of a minute rectal carcinoid less than $5 \mathrm{~mm}$ in diameter: A case report. Hepatogastroenterology 51: 1330-1332, 2004.

22. Chun HJ, Jeen YT, Park SC, Keum B, Seo YS, Um SH, Kim CD and Ryu HS: Multiple liver metastases from a rectal carcinoid tumor. Gastrointest Endosc 71: 619-620, 2010.

23. Attili F, Capurso G, Vanella G, Fuccio L, Delle Fave G, Costamagna $G$ and Larghi A: Diagnostic and therapeutic role of endoscopy in gastroenteropancreatic neuroendocrine neoplasms. Dig Liver Dis 46: 9-17, 2014.

24. De Angelis C, Brizzi RF and Pellicano R: Endoscopic ultrasonography for pancreatic cancer: Current and future perspectives. J Gastrointest Oncol 4: 220-230, 2013. 
25. Chou WC, Hung YS, Hsu JT, Chen JS, Lu CH,Hwang TL, Rau KM, Yeh KY, Chen TC and Sun CF: Chromogranin A is a reliable biomarker for gastroenteropancreatic neuroendocrine tumors in an Asian population of patients. Neuroendocrinology 95: 344-350, 2012 .

26. Belli SH, Oneto A, Aranda C, O'Connor JM, Domenichini E, Roca E, Méndez G, Bestani MC, Parma P, Giacomi N, et al: Chromogranin A as a biochemical marker for the management of neuroendocrine tumors: A multicenter study developed in Argentina. Acta Gastroenterol Latinoam 39: 184-189, 2009.

27. Gao W, Liu SM, Lu HZ, Liang J, Yuan YL and Liu XY: Analysis of clinicopathological features of intestinal neuroendocrine neoplasms. Zhonghua Zhong Liu Za Zhi 34: 450-456, 2012 (In Chinese).

28. Li AF, Li AC, Hsu CY, Li WY, Hsu HS and Chen JY: Small cell carcinomas in gastrointestinal tract: Immunohistochemical and clinicopathological features. J Clin Pathol 63: 620-625, 2010.

29. Özaslan E, Demir S, Karaca H and Güven K: Evaluation of the concordance between the stage of the disease and Ki-67 proliferation index in gastroenteropancreatic neuroendocrine tumors. Eur J Gastroenterol Hepatol 28: 836-841, 2016.

30. Wang J, Guo Y, Wang B, Bi J, Li K, Liang X, Chu H and Jiang H: Lymphatic microvessel density and vascular endothelial growth factor-C and -D as prognostic factors in breast cancer: A systematic review and meta-analysis of the literature. Mol Biol Rep 39: 11153-11165, 2012.
31. Takahashi A, Ishii G, Kinoshita T, Yoshida T, Umemura S, Hishida T, Yoh K, Niho S, Goto K, Ohmatsu H, et al: Identification of prognostic immunophenotypic features in cancer stromal cells of high-grade neuroendocrine carcinomas of the lung. J Cancer Res Clin Oncol 139: 1869-1878, 2013.

32. Watanabe M,TanakaH,Ohira M, Yoshii M, Sakurai K, Toyokawa T, Kubo N, Yamamoto A, Muguruma K, Yamashita Y, et al: Intranodal lymphangiogenesis precedes development of lymph node metastasis and accelerates progression of gastric cancer. $\mathrm{J}$ Gastrointest Surg 18: 481-490, 2014.

33. Pasquali S, van der Ploeg AP, Mocellin S, Stretch JR, Thompson JF and Scolyer RA: Lymphatic biomarkers in primary melanomas as predictors of regional lymph node metastasis and patient outcomes. Pigment Cell Melanoma Res 26: 326-337, 2013.

34. Pastushenko I, Vermeulen PB, Carapeto FJ, Van den Eynden G, Rutten A, Ara M, Dirix LY and Van Laere S: Blood microvessel density, lymphatic microvessel density and lymphatic invasion in predicting melanoma metastases: Systematic review and meta-analysis. Br J Dermatol 170: 66-77, 2014. 